\title{
Dual PI3K-BRD4 Inhibitor SF1126 Inhibits Colorectal Cancer Cell Growth in Vitro and in Vivo
}

\author{
An-cheng Qin ${ }^{a} \quad$ Ya Lib ${ }^{b}$ Li-na Zhou ${ }^{c} \quad$ Chun-gen Xing ${ }^{d} \quad$ Xing-sheng Lu ${ }^{a}$ \\ aDepartment of Hepatobiliary Surgery, Suzhou Municipal Hospital affiliated to Nanjing Medical \\ University, Suzhou, China, 'bThe Central Lab, North District, Suzhou Municipal Hospital affiliated to \\ Nanjing Medical University, Suzhou, China, 'Department of Radiotherapy and Oncology, Kunshan First \\ People's Hospital Affiliated to Jiangsu University, Kunshan, China, dDepartment of General Surgery, the \\ Second Affiliated Hospital of Soochow University, Suzhou, China
}

\section{Key Words}

Colorectal cancer • SF1126 • PI3K • BRD4 • P38

\begin{abstract}
Background/Aims: Bromodomain-containing protein 4 (BRD4) and phosphatidylinositol 3-kinase (PI3K) are key oncogenic cascades in colorectal cancer (CRC). SF1126 is a novel and potent PI3K-BRD4 dual inhibitor. Methods: CRC cells and human colon epithelial cells were treated with SF1126. Cell survival was tested by MTT and soft agar colony formation assays. Cell proliferation was tested by BrdU ELISA method. Cell apoptosis was tested by a TUNEL staining method and Histone DNA ELISA. Western blotting was utilized to test the signaling proteins. A HT-29 xenograft mice model was established to study the anti-tumor activity of SF1126 in vivo. Results: SF1126 potently inhibited the survival, proliferation, and progression of the cell cycle in an established CRC cell line (HT-29) and primary human colon cancer cells. Significant activation of apoptosis was detected in SF1126-treated CRC cells. In CRC cells, SF1126 blocked Akt-mammalian target of rapamycin (mTOR) complex $1 / 2$ signaling and downregulated BRD4 target proteins (Myc and cyclin D1). Further studies showed that SF1126 activated p38 signaling in CRC cells. In contrast, the p38 inhibitors or p38 short hairpin RNA inhibited SF1126-induced cytotoxicity and apoptosis in CRC cells. In vivo, subcutaneous administration of SF1126 significantly inhibited HT-29 xenograft tumor growth in nude mice. Conclusion: SF1126 inhibits CRC cell growth possibly by targeting PI3K-Akt-mTOR, BRD4, and p38 signaling.
\end{abstract}

A. Qin, Y. Li and L. Zhou contributed equally to this work. 


\section{Cellular Physiology Cell Physiol Biochem 2019;52:758-768

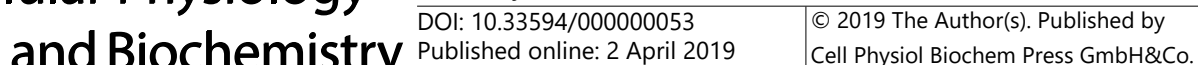 \\ Qin et al.: SF1126 Inhibits CRC Cell Growth}

\section{Introduction}

Colorectal cancer (CRC) cells with pre-existing and/or acquired resistance are resistant to conventional cytotoxic chemo-agents [1]. Genomic analyses of CRC have revealed a number of genetic mutations that critically promote tumorigenesis and tumor progression [2-4]. Among them, the phosphatidylinositol 3-kinase (PI3K)-Akt-mammalian target of rapamycin (mTOR) pathway is hyper-activated in CRC $[5,6]$. In contrast, PI3K-Akt-mTOR inhibition can efficiently inhibit CRC cell growth in vitro and in vivo $[5,6]$.

Less than $20 \%$ of patients with metastatic colorectal cancer (mCRC) will respond to current targeted therapies [2,3]. Agents that target multiple oncogenic pathways could exert more efficient anti-CRC effects [2, 3]. Bromodomain-containing protein 4 (BRD4), the most studied bromodomain and extraterminal family protein, binds to acetylated-histones, acting as a key epigenetic regulator and a novel oncogene [7-10]. BRD4 interacts with P-TEFb (positive transcription elongation factor $b$ ) and RNA polymerase II, required for transcription elongation and the expression of several oncogenes [9], including Bcl-2, Myc, and cyclin D1 [11]. Recent studies have proposed BRD4 as a potential therapeutic target of CRC [12-14].

SF1126 is an Arg-Gly-Asp (RGD)-conjugated LY294002 pro-drug, designed to exhibit enhanced solubility and binding efficiency to specific integrins in the tumor compartment [15]. SF1126 administration resulted in enhanced delivery to the tumor vasculature and tumor tissues [15]. Recent studies have shown that this compound can inhibit both PI3KAkt-mTOR and BRD4 cascades in tumor cells $[16,17]$. The present study will demonstrate that SF1126 inhibits human CRC cell growth in vitro and in vivo.

\section{Materials and Methods}

\section{Chemicals and reagents}

SF1126, LY294002, JQ1, puromycin, polybrene, SB203580, and SB239063 were purchased from SigmaAldrich (St. Louis, MO). All phosphorylation antibodies and their non-phosphorylation control antibodies were obtained from Cell Signaling Technology (Beverly, MA). The other antibodies were provided by Santa Cruz Biotechnology (Santa Cruz, CA). Cell culture reagents were provided by Gibco Co. (Suzhou, China).

\section{Cell culture}

Established HT-29 cells were cultured as previously described [18]. The primary human colon cancer cells ("pri-Can-1/2"), derived from two different colon cancer patients (men, 55 and 61 years old, stage II), as well as two primary colon epithelial cells ("pri-Epi-1/2"), were described and cultured as reported previously $[18,19]$. The experiments and protocols were approved by the institutional review board of the authors' institutions and conformed with the principles of the Declaration of Helsinki.

Methyl thiazol tetrazolium assay

After the treatments, cell viability was assessed by MTT assay. MTT optical density (OD) values at 590 nm were recorded.

\section{BrdU assay}

BrdU incorporation was tested by an ELISA kit (Cell Signaling Technology). Briefly, CRC cells with SF1126 treatment were incubated with BrdU $(10 \mu \mathrm{M})$ in the dark. Afterwards, BrdU OD values were tested at $450 \mathrm{~nm}$.

\section{Soft agar colony formation assay}

Cells after SF1126 treatment were re-suspended in agar-containing Dulbecco's modified Eagle's medium (DMEM) medium and added to 10 -cm culture dishes (10, 000 cells per dish). After 10 days of incubation, the number of colonies were fixed, stained and counted. 


\section{Cellular Physiology Cell Physiol Biochem 2019;52:758-768 \\ \begin{tabular}{l|l|l|l|l|l} 
Dol: $10.33594 / 000000053$ & 2019 \\
and The Author(s). Published by
\end{tabular} \\ Qin et al.: SF1126 Inhibits CRC Cell Growth}

Cell cycle analysis

CRC cells with SF1126 treatment were stained with propidium iodide (PI, $3 \mu \mathrm{g} / \mathrm{mL}$, Sigma-Aldrich), analyzed on a FACSCalibur machine (BD Biosciences, Franklin Lakes, N)). G0/1, S, and G2/M cell cycle percentages were recorded.

\section{Western blotting assay}

Protein lysates (30 $\mu$ g per sample) were separated by 10\% SDS-PAGE gels, which were then transferred to the polyvinylidene difluoride blots (Millipore, Burlington, MA). After blocking, the blots were incubated with specific primary and secondary (horseradish peroxidase-conjugated) antibodies. Antibody-antigen binding was detected using an enhanced chemiluminescence system. Blot intensity was quantified by ImageJ software (National Institutes of Health, Bethesda, MD).

\section{TUNEL staining assay}

Following treatment, the TUNEL (terminal deoxynucleotidyl transferase dUTP nick end labeling) In Situ Cell Death Detection Kit (Roche Diagnostics Co., Ltd., Shanghai, China) was employed to test cell apoptosis. TUNEL fluorescence intensity was measured by the Fluoroskan system [20, 21].

Caspase-3/-9 activity assay

Following SF1126 treatment, $20 \mu \mathrm{g}$ cytosolic lysates were mixed with the caspase assay buffer and the caspase-3/-9 substrate [22]. The released AFC (7-amino-4-trifluoromethyl coumarin) was tested by a spectrofluorometer with excitation wavelength of $380 \mathrm{~nm}$ and emission wavelength of $460 \mathrm{~nm}$.

\section{Histone DNA ELISA assay}

A histone DNA apoptosis ELISA kit was utilized to quantify cell apoptosis in cells with/without SF1126 treatment according to the manufacturer's instructions (Roche Diagnostics). Histone DNA ELISA OD values were recorded at $405 \mathrm{~nm}$.

Quantitative reverse transcription real-time polymerase chain reaction assay

RNA was extracted by TRIzol reagents. The First-Strand Synthesis Kit (SABiosciences, Frederick, MD) was used for cDNA synthesis. Quantitative reverse transcription real-time polymerase chain reaction (qPCR) was performed by QuantStudio 3 (Applied Biosystems) using the SYBR Green kit (Takara Bio Inc., Kusatsu, Japan). We utilized the $2^{-\Delta \Delta \mathrm{Ct}}$ method [23] to quantify targeted mRNAs, testing GAPDH $m R N A$ as the reference. The mRNA primers for cyclin D1: (F)5'-TCTACACCGACAACTCCATCCG-3' and (R)5'- TCTGGCATTTTGGAGAGGAAGTG'; for Myc, (F)5'- CCTGGTGCTCCATGAGGAGAC -3' and (R)5'-CAGACTCTGACCTTTTGCCAGG' and for GAPDH: (F)5'-GCACCGTCAAGGCTGAGAAC-3' and (R)5'TGGTGAAGACGCCAGTGGA-3' were synthesized by Genechem Co., Ltd. (Shanghai, China).

\section{p38 $\alpha$ short hairpin RNA}

HT-29 cells were seeded in the six-well tissue-culture plates. Two lentiviral short hairpin RNA (shRNA), targeting the non-overlapping sequences of human $p 38 \alpha$ [sh-p38 (-1): AAGAAGCTCTCCAGACCATTTCA and sh-p38 (-2): AAATGTGATTGGTCTGTTGGACG)], were designed, synthesized, and validated by Genechem (Shanghai, China). shRNA lentivirus was added to HT-29 cells for 24h. Stable cells were selected by puromycin $(2.5 \mu \mathrm{g} / \mathrm{mL})$ for $4-6$ passages. The expression of p38 $\alpha$ was detected by Western blotting assay.

\section{Akt1 and BRD4 double silencing}

HT-29 cells were seeded onto six-well tissue culture plates. Akt1 shRNA lentiviral particles (sc-29195-V, Santa Cruz Biotechnology) and BRD4 shRNA lentiviral particles (sc-43639-V, Santa Cruz Biotechnology) were added to HT-29 cells for $24 \mathrm{~h}$. Stable cells were selected by puromycin $(2.5 \mu \mathrm{g} / \mathrm{mL})$ for 4-6 passages. Akt1 and BRD4 knockdown in stable cells was verified by Western blotting assay. Control cells were transfected with lentiviral scramble control shRNA (Genechem). 


\section{Cellular Physiology Cell Physiol Biochem 2019;52:758-768

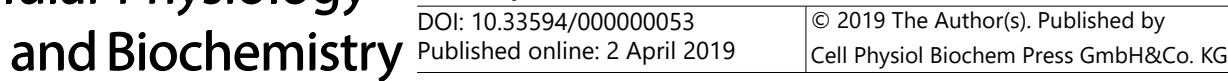 \\ Qin et al.: SF1126 Inhibits CRC Cell Growth}

\section{Xenograft assay}

Five million HT-29 cells in $100 \mu \mathrm{L}$ DMEM and $100 \mu \mathrm{L}$ Matrigel were subcutaneously injected to each nude mouse (5-6 weeks old, 18-19g). The xenografted tumors (volumes close to $100 \mathrm{~mm}^{3}$ ) were established within 20 days. The nude mice were randomly divided into three groups. The daily dose given to the two SF1126 groups was $20 \mathrm{mg} / \mathrm{kg}$ and $50 \mathrm{mg} / \mathrm{kg}$ of body weight, while an equal amount of saline was given as the vehicle control. Tumor volumes were recorded using the described formula: $\pi / 6 \times$ larger diameter $\times$ (smaller diameter) ${ }^{2}$ [18]. All studies were performed in accordance with the standards of ethical treatment approved by the Institutional Animal Care and Use Committee of the authors' institutions.

\section{Statistics analysis}

Data are expressed as the mean \pm standard deviation. Statistical analysis was performed using SPSS software (version 19.0, SPSS Inc., Chicago, IL), with $p<0.05$ taken as statistically significant.

\section{Results}

SF1126 inhibits human CRC cell survival, proliferation, and progression of the cell cycle

HT-29 cells were cultured in fetal bovine serum-containing complete medium treated with different concentrations $(0.2-10 \mu \mathrm{M})$ of the PI3K-BRD4 dual inhibitor SF1126 $[16,17]$. The MTT assay was performed to test cell viability, and the results showed that SF1126 inhibited HT-29 cell survival in a concentration- and time-dependent manner (Fig. 1A). The half maximal inhibitory concentration of SF1126 was close to 1-5 $\mu \mathrm{M}$ (72 and $96 \mathrm{~h}$ treatment, Fig. 1A). The results of soft agar colony formation assay showed that SF1126, at 1-10 $\mu \mathrm{M}$, significantly decreased the number of viable HT-29 cells (Fig. 1B). The BrdU incorporation assay was performed to test cell proliferation. As shown, SF1126 inhibited BrdU ELISA OD in HT-29 cells in a concentration-dependent manner (Fig. 1C), indicating the inhibition of proliferation. The PI-FACS assay was employed to test the cell cycle, and the quantified results showed that SF1126 (5 or $10 \mu \mathrm{M})$ treatment in HT-29 cells significantly increased the percentage of cells at the G0/1 phase (Fig. 1D), while it decreased the percentages at S- and G2/M-phases (Fig. 1D). Thus, SF1126 induced G1-S arrest in HT-29 cells.

Fig. 1. SF1126 inhibits human CRC cell survival, proliferation, and cell cycle progression. HT29 cells (A-D), primary human colon cancer cells ("pri-Can-1/2", E and F), or primary human colon epithelial cells ("priEpi-1/-2", E and F) were left untreated ("C", same in all figures) or were treated with applied concentrations (0.2-10 $\mu \mathrm{M})$ of SF1126. Cells were further cultured for the indicated times and cell survival (A, B, and E), cell proliferation ( $\mathrm{C}$ and
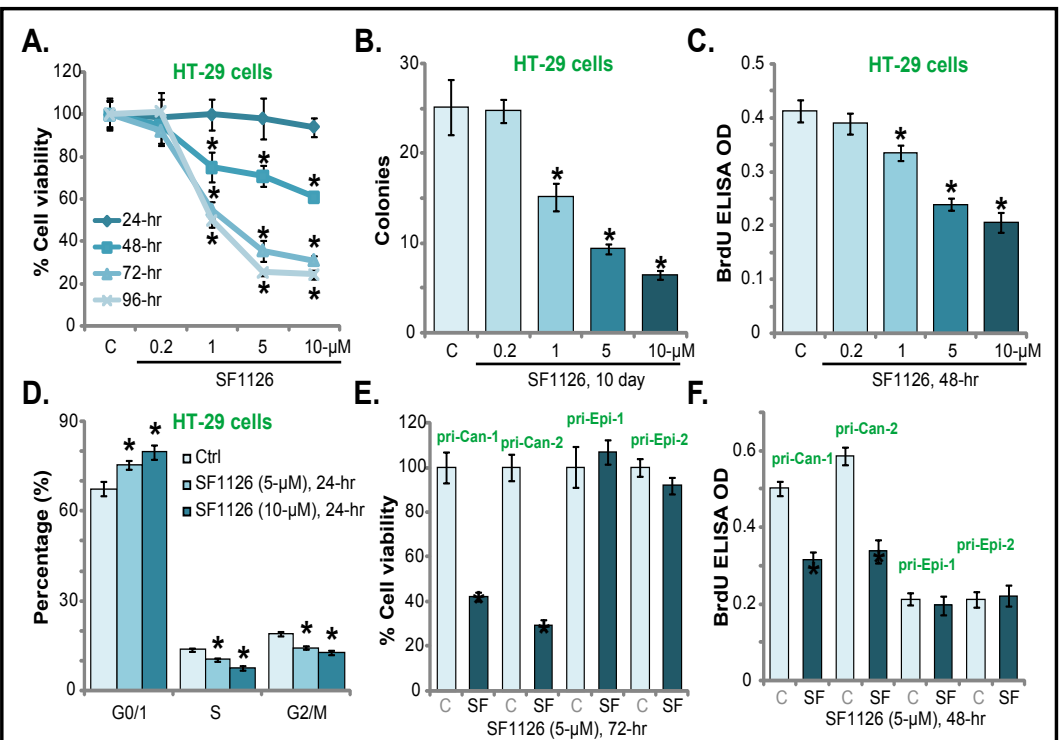

F), and cell cycle progression (D) were tested by the appropriate assays. Data are presented as the mean \pm standard deviation (in all figures). Five replicate wells/dishes were set up for each treatment (in all figures). ${ }^{*} \mathrm{p}<0.05$ vs. the "C" group. The experiments were repeated three times, with similar results obtained. 


\section{Cellular Physiology Cell Physiol Biochem 2019;52:758-768

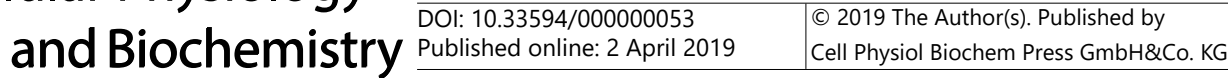 \\ Qin et al.: SF1126 Inhibits CRC Cell Growth}

The primary human colon cancer cells ("pri-Can-1/-2") and primary human colon epithelial cells ("pri-Epi-1/2") were cultured in complete medium and treated with SF1126 $(5 \mu \mathrm{M})$. As shown, the PI3K-BRD4 dual inhibitor significantly inhibited cell viability (Fig. 1E) and BrdU incorporation (Fig. 1F) in primary cancer cells. Conversely, in the colon epithelial cells, the same SF1126 treatment ( $5 \mu \mathrm{M}, 48$ and $72 \mathrm{~h}$ ) failed to affect cell survival (Fig. 1E) or proliferation (Fig. 1F). These results demonstrated that SF1126 potently inhibited human CRC cell survival, proliferation, and progression of the cell cycle.

SF1126 induces apoptosis activation in human CRC cells

The potential effect of SF1126 on cell apoptosis was examined. As shown, SF1126 dose-dependently increased the activities of caspase- 3 and caspase-9 in HT-29 cells (Fig. 2A). Furthermore, the histone-bound single strand DNA content was significantly increased in SF1126 (1-10 $\mu \mathrm{M})$-treated HT-29 cells (Fig. 2B). TUNEL fluorescence intensity was also increased in HT-29 cells following SF1126 (1-10 $\mu \mathrm{M})$ treatment (Fig. 2C). Thus, SF1126 induced apoptosis in HT-29 cells. After SF1126 (5 $\mu \mathrm{M}, 48 \mathrm{~h})$ treatment the histone-bound ssDNA content (Fig. 2D) and TUNEL intensity OD (Fig. 2E) were increased in primary human colon cancer cells ("pri-Can-1/2"). The same SF1126 treatment ( $5 \mu \mathrm{M}, 48 \mathrm{~h}$ ) failed to induce significant apoptosis in primary colon epithelial cells ("pri-Epi-1/2", Fig. 2D and E). Thus, SF1126 induced significant activation of apoptosis in human CRC cells.

\section{SF1126 inhibits Akt-mTOR and BRD4 signalings in CRC cells}

SF1126 is a dual inhibitor of PI3K and BRD4; thus, we analyzed the two signals in SF1126-treated HT-29 cells [17]. Western blotting assays were performed and the results confirmed that SF1126 inhibited the phosphorylation of Akt (Ser-473 and Thr-308) and S6K1 (Thr-389) in a dose-dependent manner, which indicated Akt-mTORC1/2 inhibition (Fig. 3A). Akt-S6K1 phosphorylation was also blocked in SF1126 (5 $\mu \mathrm{M}, 2 \mathrm{~h})$-treated primary human colon cancer cells ("pri-Can-1/2", Fig. 3B and C). Total Akt and S6K1 expression levels were unchanged (Fig. 3A-C).

Myc $[10,24,25]$ and cyclin D1 [26, 27] are key BRD4 targets. qPCR assay results confirmed that Myc mRNA (Fig. 3D) and cyclin D1 mRNA (Fig. 3E) levels were significantly decreased in SF1126 (1-10 $\mu \mathrm{M})$-treated HT-29 cells. Myc and cyclin D1 proteins were also downregulated (Fig. 3F). In the human colon cancer cells ("pri-Can-1/2"), SF1126 (5 $\mu \mathrm{M})$ also induced Myc and cyclin D1 downregulation (Fig. 3G and H). Therefore,SF1126 inhibited Akt-mTOR and BRD4 signaling in CRC cells.

Fig. 2. SF1126 induces apoptosis activation in human CRC cells. HT-29 cells (A-C), primary human colon cancer cells ("pri-Can-1/2", $\mathrm{D}$ and $\mathrm{E}$ ), or primary human colon epithelial cells ("pri-Epi-1/-2", D and E) were treated with applied concentrations of SF1126. Cells were further cultured for indicated times, and caspase activation and the induction of apoptosis were tested by the assays described in the text $(\mathrm{A}-\mathrm{E}) .{ }^{*} \mathrm{p}<0.05$ vs. the " $C$ " group. The experiments were repeated three times, with similar results obtained.

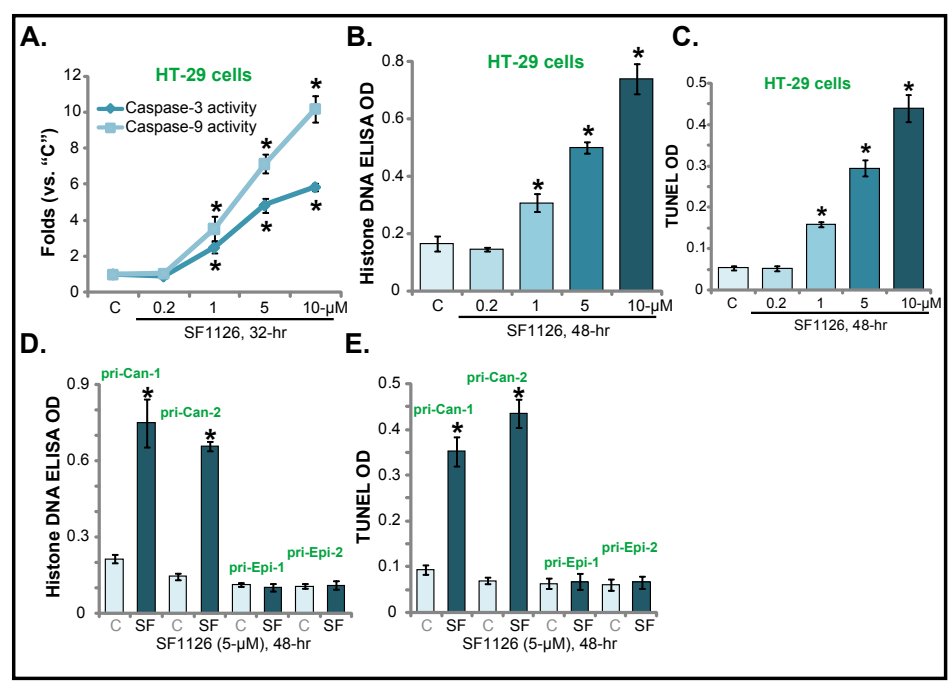




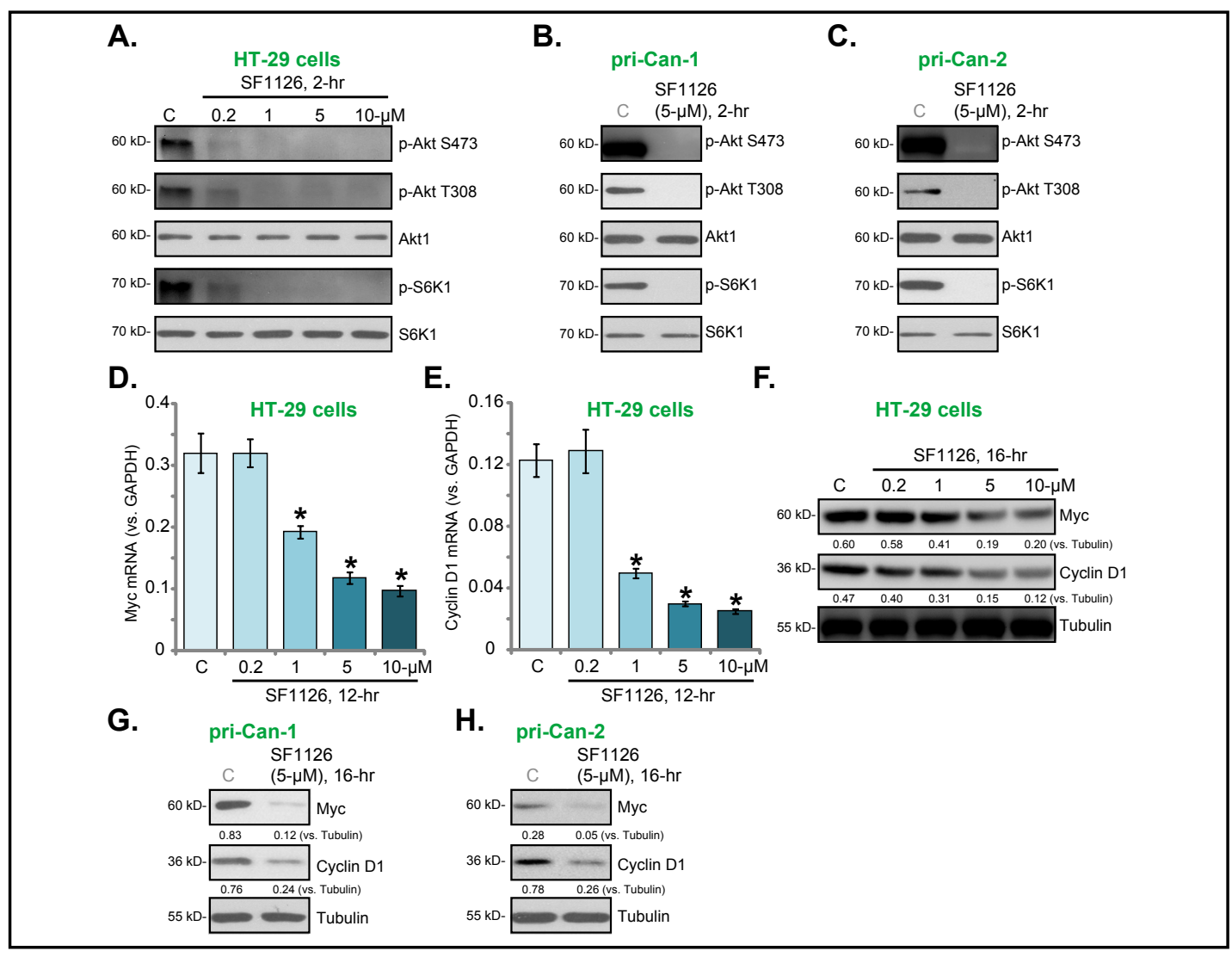

Fig. 3. SF1126 inhibits Akt-mTOR and BRD4 signalings in CRC cells. HT-29 cells (A-F) or primary human colon cancer cells ("pri-Can-1/2", G and H) were treated with applied concentrations (0.2-10 $\mu \mathrm{M})$ of SF1126. Cells were further cultured for the indicated times, and the expression of the listed mRNA and proteins in total cell lysates were tested by qPCR and Western blotting assays, respectively. The total gray area in each band was quantified and normalized to tubulin $(\mathrm{F}-\mathrm{H}) .{ }^{*} \mathrm{p}<0.05$ vs. the "C" group (D and E). The experiments were repeated three times, with similar results obtained.

\section{SF1126 is more potent than other PI3K-BRD4 inhibitors in killing CRC cells}

We compared the anti-CRC cell activity of SF1126 with other known PI3K or BRD4 inhibitors. JQ1 is a well-known BRD4 inhibitor and LY294002 is a pan PI3K-Akt-mTOR inhibitor [28]. In HT-29 cells, treatment with JQ1 or LY294002 induced a moderate reduction in viability (Fig. 4A) and apoptosis (Fig. 4B), but this was less potent than that with SF1126 treatment (Fig. 4A and B). SF1126 killed HT-29 cells more efficiently than JQ1 plus LY294002 combined (Fig. 4A and B). Similarly, in the primary human colon cancer cells ("pri-Can-2"), JQ1 plus LY294002 co-treatment was less effective than SF1126 in inducing cell death and apoptosis (Fig. 4C and D). These results suggest that other mechanisms, besides BRD4 and PI3K-Akt-mTOR inhibition, are also responsible for SF1126-induced anti-CRC cell activity.

The shRNA method was utilized to silence Akt1 and BRD4 in HT-29 cells. As described, lentiviral Akt1 shRNA and lentiviral BRD4 shRNA were added to HT-29 cells. Stable cells were established by puromycin selection, where Akt1 and BRD4 were almost completely silenced ("sh-Akt1+BRD4" cells, Fig. 4E). When compared with the control cells ("sh-C" cells), the sh-Akt1+BRD4 cells presented with decreased viability and increased cell apoptosis (Fig. 4F and G). Importantly, SF1126 could still induce cytotoxicity and apoptosis in "sh-Akt1+BRD4" cells, whereas JQ1 plus LY294002 combination treatment was ineffective (Fig. 4F and G). There results further confirmed that Akt1-BRD4-independent mechanisms were involved in SF1126-induced cytotoxicity in CRC cells. 


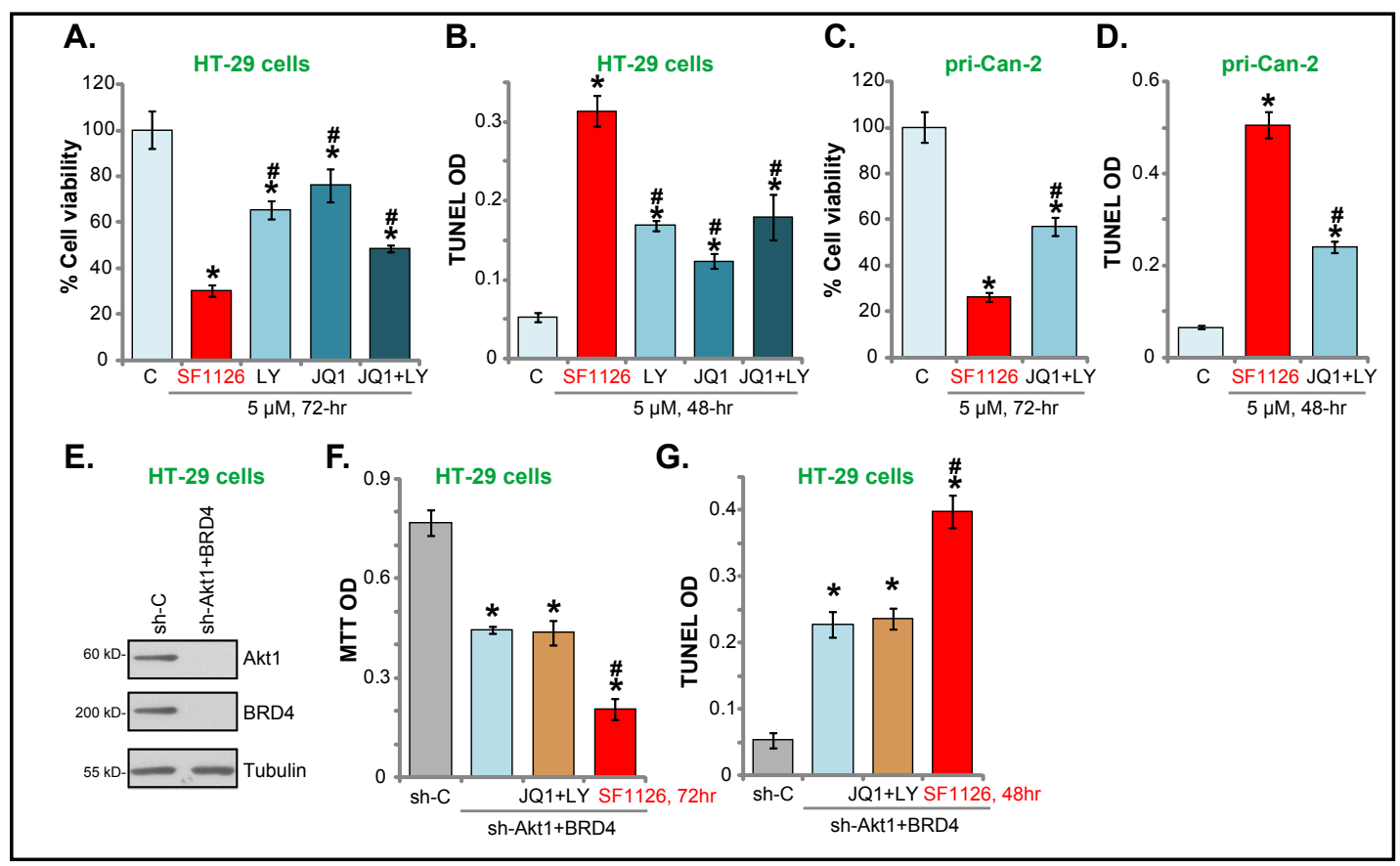

Fig. 4. SF1126 is more potent than other PI3K-BRD4 inhibitors in killing CRC cells. HT-29 cells (A and B) or primary human colon cancer cells ("pri-Can-2", C and D) were treated with $5 \mu \mathrm{M}$ of SF1126, LY294002 ("LY"), JQ1, or LY plus JQ1. Cell viability and apoptosis were tested by the MTT assay (A and C) and TUNEL fluorescence intensity assay (B and D), respectively. Stable HT-29 cells, with Akt1 shRNA plus BRD shRNA ["sh-Akt1+BRD4"] or scramble control shRNA ("sh-C"), were treated with/without SF1126 (5 $\mu \mathrm{M})$ or LY294002 $(5 \mu \mathrm{M})$ plus JQ1 $(5 \mu \mathrm{M})$ for the indicated times; expression of the listed proteins are shown (E). Cell viability (F) and apoptosis (G) were tested similarly. ${ }^{*} \mathrm{p}<0.05$ vs. the "C" group (A-D). ${ }^{*} \mathrm{p}<0.05$ vs. "SF1126" treatment (A-D). *p< 0.05 vs. "sh-C" cells (F and G). ${ }^{*}$ p 0.05 vs. untreated "sh-Akt1+BRD4" cells (F and $\mathrm{G})$. The experiments were repeated three times, with similar results obtained.

\section{SF1126 activates $p 38$ in CRC cells}

Activation of p38 can promote human CRC cell apoptosis [29, 30]. In the present study, we showed that SF1126 activated p38 signaling in HT-29 cells, as evidenced by increased phosphorylation of apoptosis signal-regulating kinase 1 (ASK1) and p38 (Fig. 5A). JQ1 plus LY294002 co-treatment failed to induce ASK1-p38 activation (Fig. 5A), suggesting that SF1126-induced p38 activation was independent of PI3K-BRD4 inhibition. Two p38 inhibitors (SB203580 and SB239063) inhibited, but did not reverse, SF1126-induced HT-29 cell viability reduction (Fig. 5B) and apoptosis (Fig. 5C). Thus, p38 activation participated in SF1126-induced cytotoxicity in HT-29 cells.

To further support the hypothesis, two lentiviral shRNAs that target non-overlapping sequences of p38 ["sh-p38 (-1)/(-2)"] were individually transfected into HT-29 cells. Stable cells were established, with depleted p38 via puromycin selection( Fig. 5D). SF1126-induced p38 activation was blocked in HT-29 cells with p38 shRNA (Fig. 5D). Consequently, SF1126induced cytotoxicity (Fig. 5E) and apoptosis (Fig. 5F) were attenuated, further supporting the evidence that p38 activation contributed to SF1126-induced cytotoxicity in CRC cells. Notably, neither SB203580 nor p38 shRNA ["sh-p38 (-1)"] had an effect on JQ1 plus LY294002 co-treatment-induced HT-29 cell death (Fig. 5G), suggesting that p38 activation is an unique action of SF1126. In primary human colon cancer cells ("pri-Can-2"), SF1126 (5 $\mu \mathrm{M}, 2 \mathrm{~h}$ ) provoked p38 activation (Fig. 5H). The p38 inhibitors SB203580 and SB239063 attenuated SF1126-induced cytotoxicity in primary cancer cells (Fig. 5I). 
Fig. 5. SF1126 activates p38 signaling in CRC cells. HT-29 cells were treated with $5 \mu \mathrm{M}$ SF1126 or LY294002 ("LY") plus JQ1 for $2 \mathrm{~h}$. Expression of listed proteins in total cell lysates were tested (A). HT-29 cells were pretreated for $30 \mathrm{~min}$ with $5 \mu \mathrm{M}$ of SB203580 or SB239063, followed by the SF1126 treatment. Cell viability and apoptosis were tested by the MTT assay (B) and TUNEL fluorescence intensity assay (C), respectively. Stable HT-29 cells, with p38 shRNA ["sh-p38 $(-1) /(-2)$ "] or scramble control shRNA ("sh-C"), were treated with $5 \mu \mathrm{M}$ of SF1126 for $2 \mathrm{~h}$; listed proteins were shown (D). Cells were also cultured for 48 or $72 \mathrm{~h}$, and cell viability (E)

and apoptosis (F) were tested. HT-29 cells, pretreated with SB203580 (5 $\mu \mathrm{M})$ or with p38 shRNA ["sh-p38 $\left.(-1)^{\prime \prime}\right]$, were treated with $5 \mu \mathrm{M}$ LY plus JQ1 for $72 \mathrm{~h}$, and cell viability was tested (G). The primary human colon cancer cells ("pri-Can-2") were treated with $5 \mu \mathrm{M} \mathrm{SF1126}$ for $2 \mathrm{~h}$; expression of listed proteins are shown (H). Primary cancer cells were pretreated for $30 \mathrm{~min}$ with $5 \mu \mathrm{M}$ SB203580 or SB239063, followed by $5 \mu \mathrm{M}$ of SF1126 treatment for $72 \mathrm{~h}$; cell viability was tested (I). "DMSO" stands for $0.1 \%$ dimethyl sulfoxide. *p $<0.05$ vs. the "C" group. " $\mathrm{p}<0.05$ vs. "SF1126" treatment in the "DMSO" group or in the "sh-C" group. The experiments were repeated three times, with similar results obtained.

\section{Antitumor effect of SF1126 against HT-29 xenograft in mice}

Finally, we tested the anti-tumor activity of SF1126 in vivo. A significant number of HT-29 cells (five million cells per mouse) were inoculated (via subcutaneous injection) to the flanks of the nude mice. The volumes of the xenografts reached $100 \mathrm{~mm}^{3}$ within 20 days when the SF1126 administration commenced ("Day0"). By recording tumor volumes, we showed that SF1126, when injected subcutaneously at 20 or $50 \mathrm{mg} / \mathrm{kg}$ daily [17], significantly inhibited HT-29 xenograft growth in the mice (Fig. 6A). The estimated daily tumor growth, calculated by ([tumor volume at Day36] - [tumor volume at Day0])/36, was significant lower after SF1126 administration (Fig. 6B). At Day36, all tumors were isolated and weighed. The results showed that tumors in SF1126-treated mice were significantly lighter than those of the vehicle control mice (Fig. 6C). SF1126 inhibited tumor growth, with no evidence of toxicity, as measured by weight loss (Fig. 6D). 


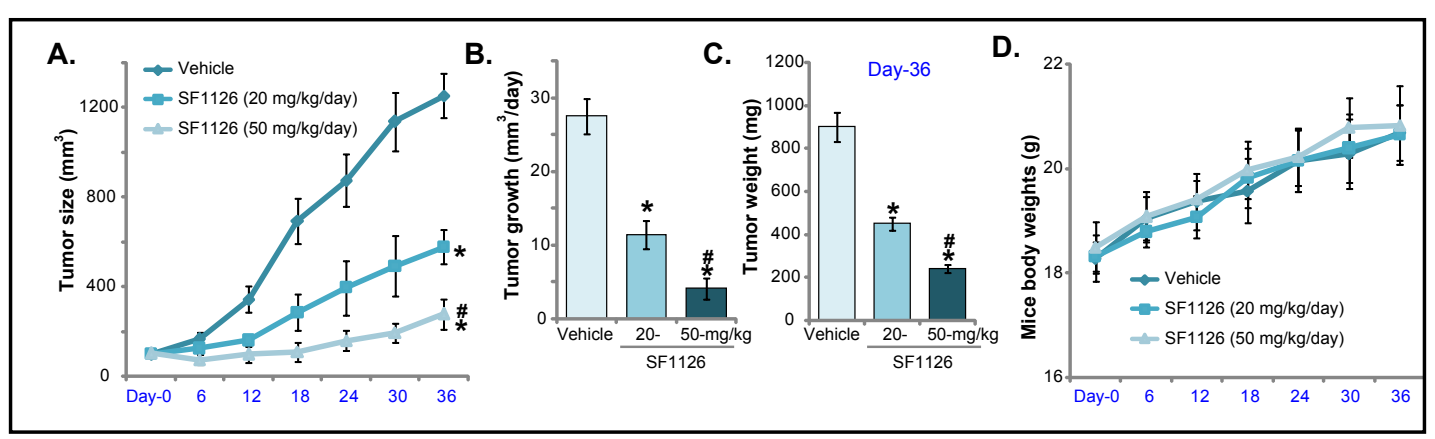

Fig. 6. Antitumor effect of SF1126 against HT-29 xenograft in nude mice. Five million HT-29 cells were injected subcutaneously in each nude mouse. After 20 days, HT-29 tumor-bearing mice were randomly divided in to three groups ( $\mathrm{n}=10$ per group), treated daily with SF1126 (20 or 50-mg/kg body weight, subcutaneous) or saline vehicle control ("Vehicle"). Tumor volume (A) and mice body weight (D) were recorded every 6 days. The estimated daily tumor growth (in $\mathrm{mm}^{3}$ per day, B) and tumor weights at Day36 (C) are shown. ${ }^{*} \mathrm{p}<0.05$ vs. the "Vehicle" group (A-C). ${ }^{\#} \mathrm{p}<0.05$ vs. the $20 \mathrm{mg} / \mathrm{kg}$ SF1126 group (A-C).

\section{Discussion}

Agents targeting the PI3K-Akt-mTOR pathway should effectively treat CRC when combined with other targeted therapies. SF1126 is composed of LY294002 conjugated to a RGD-targeting peptide. This increases the solubility and binding to integrins on the tumor vasculature. The pro-drug enhances tumor delivery of the active inhibitor, improving antitumor efficacy and tolerability $[15,31]$. Recent studies have shown that SF1126 also blocks the BRD4 pathway $[16,17]$. Our studies show that SF1126 potently inhibited survival, proliferation, and progression of the cell cycle in an established CRC cell line (HT-29) and primary human colon cancer cells. Significant activation of apoptosis was detected in SF1126-treated CRC cells. The dual inhibitor was non-cytotoxic to normal colon epithelial cells. SF1126 blocked Akt-mTORC1/2 signaling activation and downregulated key BRD4 targets (Myc and Cyclin D1) in CRC cells. In vivo, subcutaneous administration of SF1126 at well-tolerated doses significantly inhibited HT-29 xenograft tumor growth in mice.

Notably, SF1126 was more potent in inhibiting human CRC cells than the BRD4 specific inhibitor (JQ1) or the PI3K-Akt-mTOR pan inhibitor (LY294002). SF1126 was also more efficient than JQ1 and LY294002 combined in killing CRC cells. It even provoked cytotoxicity in Akt1- and BRD4-double-silenced HT-29 cells. These results implied the involvement of PI3KBRD4-independent mechanisms in mediating SF1126's actions. Indeed, SF1126 activated p38 signaling in CRC cells. Conversely, pharmacological p38 inhibition or p38 silencing by targeted shRNAs inhibited SF1126-induced cytotoxicity and apoptosis in CRC cells.

Also important was that JQ1 plus LY294002 combination treatment failed to induce p38 activation. Furthermore, p38 inhibition or silencing had no significant effect on JQ1 plus LY294002 co-treatment-induced cytotoxicity. These results indicate that p38 activation is an unique action of SF1126. Previous studies have shown that p38 inactivated cyclin D1-CDK4 pathway to induce nuclear translocation of RelA, which promoted CRC cell apoptosis [29]. Furthermore, p38 activation negatively regulated survivin and gamma-H2AX in CRC cells, thus facilitating cell apoptosis [30]. Therefore, concurrent activation of the p38 pathway could be one key explanation of the superior anti-CRC activity by SF1126.

\section{Conclusion}

Here, we show that SF1126 induced PI3K-Akt-mTOR and BRD4 blockage as well asp38 activation, causing significant CRC cell inhibition. SF1126 should be further tested as a promising and novel anti-CRC agent. 


\section{Cellular Physiology Cell Physiol Biochem 2019;52:758-768

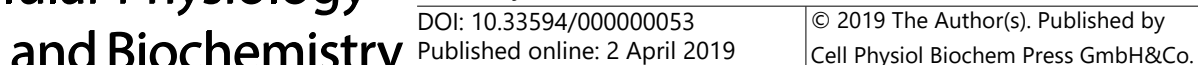 \\ Qin et al.: SF1126 Inhibits CRC Cell Growth}

\section{Acknowledgements}

This work was generously supported by the Suzhou Key Medical Center (LCZX201505), the Suzhou Science and Technology Development Program (SZS201618), the National Natural Science Foundation of China (81672970), the Second Affiliated Hospital of Soochow University Preponderant Clinical Discipline Group Project Funding and the Suzhou Science and Technology Advisory Plan (SYSD2018120).

\section{Disclosure Statement}

The authors declare that no conflicts of interest exist.

\section{References}

1 Liscovitch M, Lavie Y: Cancer multidrug resistance: a review of recent drug discovery research. IDrugs 2002;5:349-355.

2 Hubbard JM, Grothey A: Colorectal cancer in 2014: Progress in defining first-line and maintenance therapies. Nat Rev Clin Oncol 2015;12:73-74.

3 Schmoll HJ, Stein A: Colorectal cancer in 2013: Towards improved drugs, combinations and patient selection. Nat Rev Clin Oncol 2014;11:79-80.

$>4$ Kerr D: Clinical development of gene therapy for colorectal cancer. Nat Rev Cancer 2003;3:615-622.

5 Francipane MG, Lagasse E: mTOR pathway in colorectal cancer: an update. Oncotarget 2014;5:49-66. Pandurangan AK: Potential targets for prevention of colorectal cancer: a focus on PI3K/Akt/mTOR and Wnt pathways. Asian Pac J Cancer Prev 2013;14:2201-2205.

7 Iftner T, Haedicke-Jarboui J, Wu SY, Chiang CM: Involvement of Brd4 in different steps of the papillomavirus life cycle. Virus Res 2017;231:76-82.

8 Bachu M, Dey A, Ozato K: Chromatin Landscape of the IRF Genes and Role of the Epigenetic Reader BRD4. J Interferon Cytokine Res 2016;36:470-475.

9 Devaiah BN, Singer DS: Two faces of brd4: mitotic bookmark and transcriptional lynchpin. Transcription 2013;4:13-17.

10 Zuber J, Shi J, Wang E, Rappaport AR, Herrmann H, Sison EA, Magoon D, Qi J, Blatt K, Wunderlich M, Taylor MJ, Johns C, Chicas A, Mulloy JC, Kogan SC, Brown P, Valent P, Bradner JE, Lowe SW, Vakoc CR: RNAi screen identifies Brd4 as a therapeutic target in acute myeloid leukaemia. Nature 2011;478:524-528.

11 Wu X, Liu D, Gao X, Xie F, Tao D, Xiao X, Wang L, Jiang G, Zeng F: Inhibition of BRD4 Suppresses Cell Proliferation and Induces Apoptosis in Renal Cell Carcinoma. Cell Physiol Biochem 2017;41:1947-1956.

12 Togel L, Nightingale R, Chueh AC, Jayachandran A, Tran H, Phesse T, Wu R, Sieber OM, Arango D, Dhillon AS, Dawson MA, Diez-Dacal B, Gahman TC, Filippakopoulos P, Shiau AK, Mariadason JM: Dual Targeting of Bromodomain and Extraterminal Domain Proteins, and WNT or MAPK Signaling, Inhibits c-MYC Expression and Proliferation of Colorectal Cancer Cells. Mol Cancer Ther 2016;15:1217-1226.

13 McCleland ML, Mesh K, Lorenzana E, Chopra VS, Segal E, Watanabe C, Haley B, Mayba O, Yaylaoglu M, Gnad F, Firestein R: CCAT1 is an enhancer-templated RNA that predicts BET sensitivity in colorectal cancer. J Clin Invest 2016;126:639-652.

14 Hu Y, Zhou J, Ye F, Xiong H, Peng L, Zheng Z, Xu F, Cui M, Wei C, Wang X, Wang Z, Zhu H, Lee P, Zhou M, Jiang B, Zhang DY: BRD4 inhibitor inhibits colorectal cancer growth and metastasis. Int J Mol Sci 2015;16:19281948.

15 Garlich JR, De P, Dey N, Su JD, Peng X, Miller A, Murali R, Lu Y, Mills GB, Kundra V, Shu HK, Peng Q Durden DL: A vascular targeted pan phosphoinositide 3-kinase inhibitor prodrug, SF1126, with antitumor and antiangiogenic activity. Cancer Res 2008;68:206-215.

16 Erdreich-Epstein A, Singh AR, Joshi S, Vega FM, Guo P, Xu J, Groshen S, Ye W, Millard M, Campan M, Morales G, Garlich JR, Laird PW, Seeger RC, Shimada H, Durden DL: Association of high microvessel alphavbeta3 and low PTEN with poor outcome in stage 3 neuroblastoma: rationale for using first in class dual PI3K/BRD4 inhibitor, SF1126. Oncotarget 2017;8:52193-52210. 


\section{Cellular Physiology Cell Physiol Biochem 2019;52:758-768 \begin{tabular}{c|c|c|} 
Dol: 10.33594/000000053 & 02019 The Author(s). Published by \\
and Biochemistry Publised &
\end{tabular} and Biochemistry Published online: 2 April 2019 Cell Physiol Biochem Press GmbH\&Co. KG \\ Qin et al.: SF1126 Inhibits CRC Cell Growth}

17 Singh AR, Joshi S, Burgoyne AM, Sicklick JK, Ikeda S, Kono Y, Garlich JR, Morales GA, Durden DL: Single Agent and Synergistic Activity of the "First-in-Class" Dual PI3K/BRD4 Inhibitor SF1126 with Sorafenib in Hepatocellular Carcinoma. Mol Cancer Ther 2016;15:2553-2562.

18 Lu XS, Qiao YB, Li Y, Yang B, Chen MB, Xing CG: Preclinical study of cinobufagin as a promising anticolorectal cancer agent. Oncotarget 2017;8:988-998.

19 Li JP, Huang ZJ, Lu XS, Zhou YC, Shao Y, He XP, Chen SR, Wang DD, Qin LS, Sun WH: Pre-clinical characterization of PKC412, a multi-kinase inhibitor, against colorectal cancer cells. Oncotarget 2016;7:77815-77824.

20 Yang YL, Ji C, Bi ZG, Lu CC, Wang R, Gu B, Cheng L: Deguelin induces both apoptosis and autophagy in cultured head and neck squamous cell carcinoma cells. PLoS One 2013;8:e54736.

21 Li KR, Zhang ZQ Yao J, Zhao YX, Duan J, Cao C, Jiang Q: Ginsenoside Rg-1 protects retinal pigment epithelium (RPE) cells from cobalt chloride (CoCl2) and hypoxia assaults. PLoS One 2013;8:e84171.

22 Jiang SJ, Wang S: Dual targeting of mTORC1 and mTORC2 by INK-128 potently inhibits human prostate cancer cell growth in vitro and in vivo. Tumour Biol 2015;36:8177-8184.

23 Liu T, Wu C, Weng G, Zhao Z, He X, Fu C, Sui Z, Huang SX: Bufalin Inhibits Cellular Proliferation and Cancer Stem Cell-Like Phenotypes via Upregulation of MiR-203 in Glioma. Cell Physiol Biochem 2017;44:671-681.

24 Andrews FH, Singh AR, Joshi S, Smith CA, Morales GA, Garlich JR, Durden DL, Kutateladze TG: Dual-activity PI3K-BRD4 inhibitor for the orthogonal inhibition of MYC to block tumor growth and metastasis. Proc Natl Acad Sci U S A 2017;114:E1072-E1080.

25 Ambrosini G, Sawle AD, Musi E, Schwartz GK: BRD4-targeted therapy induces Myc-independent cytotoxicity in Gnaq/11-mutatant uveal melanoma cells. Oncotarget 2015;6:33397-33409.

26 Qiu H, Li J, Clark LH, Jackson AL, Zhang L, Guo H, Kilgore JE, Gehrig PA, Zhou C, Bae-Jump VL: JQ1 suppresses tumor growth via PTEN/PI3K/AKT pathway in endometrial cancer. Oncotarget 2016;7:6680966821.

27 Zhang L, Tong Y, Zhang X, Pan M, Chen S: Arsenic sulfide combined with JQ1, chemotherapy agents, or celecoxib inhibit gastric and colon cancer cell growth. Drug Des Devel Ther 2015;9:5851-5862.

28 Brunn GJ, Williams J, Sabers C, Wiederrecht G, Lawrence JC, Jr., Abraham RT: Direct inhibition of the signaling functions of the mammalian target of rapamycin by the phosphoinositide 3-kinase inhibitors, wortmannin and LY294002. EMBO J 1996;15:5256-5267.

29 Thoms HC, Dunlop MG, Stark LA: p38-mediated inactivation of cyclin D1/cyclin-dependent kinase 4 stimulates nucleolar translocation of RelA and apoptosis in colorectal cancer cells. Cancer Res 2007;67:1660-1669.

30 Hsiao PW, Chang CC, Liu HF, Tsai CM, Chiu TH, Chao JI: Activation of p38 mitogen-activated protein kinase by celecoxib oppositely regulates survivin and gamma-H2AX in human colorectal cancer cells. Toxicol Appl Pharmacol 2007;222:97-104.

31 Ozbay T, Durden DL, Liu T, O’Regan RM, Nahta R: In vitro evaluation of pan-PI3-kinase inhibitor SF1126 in trastuzumab-sensitive and trastuzumab-resistant HER2-over-expressing breast cancer cells. Cancer Chemother Pharmacol 2010;65:697-706. 